ARTICLE

\title{
Mechanism and inhibition of Streptococcus pneumoniae IgA1 protease
}

\author{
Zhiming Wang ${ }^{1,5}$, Jeremy Rahkola ${ }^{2,5}$, Jasmina S. Redzic', Ying-Chih Chi ${ }^{3}$, Norman Tran ${ }^{4}$, Todd Holyoak (D) 4 , \\ Hongjin Zheng (i) ${ }^{1 凶}$, Edward Janoff(i) ${ }^{2 凶} \&$ Elan Eisenmesser (i) ${ }^{1 \times}$
}

Opportunistic pathogens such as Streptococcus pneumoniae secrete a giant metalloprotease virulence factor responsible for cleaving host $\lg A 1$, yet the molecular mechanism has remained unknown since their discovery nearly 30 years ago despite the potential for developing vaccines that target these enzymes to block infection. Here we show through a series of cryo-electron microscopy single particle reconstructions how the Streptococcus pneumoniae IgA1 protease facilitates IgA1 substrate recognition and how this can be inhibited. Specifically, the Streptococcus pneumoniae IgA1 protease subscribes to an active-site-gated mechanism where a domain undergoes a $10.0 \AA$ movement to facilitate cleavage. Monoclonal antibody binding inhibits this conformational change, providing a direct means to block infection at the host interface. These structural studies explain decades of biological and biochemical studies and provides a general strategy to block Streptococcus pneumoniae IgA1 protease activity to potentially prevent infection.

\footnotetext{
${ }^{1}$ Department of Biochemistry and Molecular Genetics, School of Medicine, University of Colorado Denver, School of Medicine, Aurora, CO 80045, USA ${ }^{2}$ Mucosal and Vaccine Research Program Colorado, Division of Infectious Disease, University of Colorado Denver School of Medicine and Denver Veterans Affairs Medical Center, Aurora, CO 80045, USA. ${ }^{3}$ Cryo-EM Center, Vagelos College of Physicians and Surgeons, Columbia University Irving Medical Center, New York, NY 10032, USA. ${ }^{4}$ Department of Biology, University of Waterloo, Waterloo, ON, Canada N2L 3G1. ${ }^{5}$ These authors contributed equally: Zhiming Wang, Jeremy Rahkola. ${ }^{凶}$ email: Hongjin.Zhing@ucdenver.edu; Edward.Janoff@cuanschutz.edu; Elan.Eisenmesser@ucdenver.edu
} 
S treptococcus pneumoniae (S. pneumoniae) is a leading cause of bacterial pneumonia and meningitis in children and adults worldwide ${ }^{1-4}$. According to the World Health Organization (WHO), S. pneumoniae remains a "major global public health problem". Polysaccharide-based vaccines have proven effective against invasive $S$. pneumoniae, but less so against mucosal infections, and these vaccines target only a subset of the known serotypes ${ }^{5}$. Thus, employing a more widely shared protein virulence factor such as the $S$. pneumoniae IgA1 protease (IgA1P) as a vaccine target has been advocated, as it is present in all pathogenic strains and active at the respiratory mucosa ${ }^{6-11}$.

S. pneumoniae IgA1P is the prototypical member of the M26 class of bacterial metalloproteases ${ }^{12}$, which share virtually no sequence homology to previously characterized proteins ${ }^{9}$. Other opportunistic pathogens that secrete similar IgA1 metalloproteases include Streptococcus oralis and Streptococcus sanguinis ${ }^{9}$. The mature forms of these enzymes comprise nearly 2000 amino acids and are covalently linked to their bacterial cell surface by sortase $\mathrm{A}^{13}$. Beyond the metalloprotease class of IgA1Ps, there are two other structurally distinct IgA1P classes that include serine proteases $^{14}$ and cysteine proteases ${ }^{15}$, illustrating the evolutionary need to converge upon mechanisms of IgA1 cleavage in order to thwart the initial host immune response. All classes of bacterial IgA1Ps cleave host IgA1 within the heavy-chain (HC) hinge region, the linker connecting the IgA1 constant fragment $(\mathrm{Fc})$ to its antigen binding region (Fab) (Fig. 1a), leaving the light chain (LC) intact while separating the Fc and Fab. The shorter linker within IgA2 and IgGs renders them inaccessible to IgA1P cleavage $^{16}$. Proteolysis by IgA1Ps serves two purposes: to prevent phagocyte killing by decoupling the IgA1 Fc recognized by neutrophils from the pathogen-recognizing $\mathrm{Fab}^{6,17}$ and to coat the bacterial surface with non-functional Fab fragments to effectively shield it from immune surveillance ${ }^{18,19}$. Despite the discovery of the metalloprotease class of bacterial virulence factors 30 years $\operatorname{ago}^{20}$, their structure and mechanism of substrate engagement has remained unknown until now.

In this work, we use cryo-electron microscopy (cryo-EM) to elucidate the structure of the $S$. pneumoniae IgA1P catalytic region alone and in complex with both its IgA1 substrate and a neutralizing monoclonal antibody $(\mathrm{mAb})$, thereby addressing the molecular basis of substrate recognition and enzyme inhibition.

\section{Results}

The high-resolution structure of the IgA1P catalytic region. In order to identify the $S$. pneumoniae IgA1P catalytic domain, we engineered several constructs based on our previous results of limited proteolysis on the full, mature IgA1P (residues 154-1963, UniProt accession Q59947; NCBI accession WP_000417171 that corresponds to the common D39 and R6 strains) ${ }^{21,22}$. Only constructs that began at or prior to residue 665 were accessible to enzymatic cleavage of the MBP tag by thrombin (Supplementary Fig. 1a) and there was an observed reduction in IgA1-cleavage for shorter constructs (Supplementary Fig. 1b). Thus, we focused our cryo-EM studies on a construct of IgA1P spainning residues 665-1963 that could be excised from its MBP tag and had comparable cleavage to the full-length IgA1P (Fig. 1b).

The 3D cryo-EM reconstruction of the S. pneumoniae IgA1P (residues 665-1963) resulted in a $3.8 \AA$ resolution map (resolution shells are shown in Fig. $1 \mathrm{c}$ and structural data parameters and refinement statistics are presented in Supplementary Fig. 2 and Supplementary Table 1). The overall structure reveals that $S$. pneumoniae IgA1P is a multi-domain enzyme (Fig. 1d), broadly comprised of N-terminal (NTD; residues 665-1070), middle (MD; 1071-1611), and C-terminal domains (CTD; 1612-1963). The NTD can be further divided into a small subdomain (residues 665-769) attached by a long linker to a $\beta$-helix (residues 781-1070), a common structural motif found in several bacterial proteins $^{23}$. A defining feature of this $S$. pneumoniae $\beta$-helix is both its relatively small size and the lack of protruding secondary structure elements from within the domain itself. For example, the $\beta$-helix of the functionally similar but structurally unrelated serine IgA1P from Haemophilus influenzae is about twice as long and has multiple domains that emanate from and return to the $\beta$ helical core ${ }^{24}$. The IgA1P MD and CTD have no structural similarity to any known protein to date, as indicated by the lack of structural similarity using DALI searches. The active site is formed between the MD and CTD domains that bifurcate the $\mathrm{Zn}$ coordinating residues of the HEMTH motif (residues 1604-1608 in the MD) and a downstream E (E1628 in the CTD) (Fig. 1d, e). Despite the unique structural folds of the IgA1P MD and CTD, the S. pneumoniae IgA1P active site comprises catalytically important residues within similar positions to that of the prototypical metalloprotease, thermolysin (Fig. 1f), which supports a conserved catalytic mechanism that is consistent with our previous biochemical studies ${ }^{21}$. Specifically, three side chains from IgA1P H1604, H1608, and the downstream E1628 coordinate the $\mathrm{Zn}$ atom and are homologous with thermolysin residues H142, H146, and E166, respectively. The $\mathrm{Zn}$ both polarizes the carbonyl group of the scissile peptide bond and activates the nucleophilic water molecule that is deprotonated by a fourth residue, E1605 (E143 in thermolysin) ${ }^{25}$.

Trapping an active IgA1P/IgA1 complex. In order to trap the $S$. pneumoniae IgA1P in complex with its IgA1 substrate, several challenges were overcome. We engineered an active-site mutant in the context of IgA1P 665-1963 that removes one of the $\mathrm{Zn}$ coordinating residues, referred to as IgA1P-E1605A. While this mutation abrogates catalysis under catalytic concentrations of the enzyme (Supplementary Fig. 1b), catalysis was still observed during incubation at stoichiometric concentrations of the IgA1PE1605A/IgA1 complex (Supplementary Fig. 1c). As EDTA was found to slow catalysis (Supplementary Fig. 1c), the IgA1PE1605A/IgA1 complex was purified for cryo-EM studies in the presence of EDTA (Supplementary Fig. 1d). A cryo-EM 3D reconstruction of the complex resulted in a $3.5 \AA$ resolution map (Resolution shells are shown in Fig. 2a and structural data parameters and refinement statistics in Supplementary Fig. 2 and Supplementary Table 1), which facilitated fits of the individual domains with subsequent refinement.

The high-resolution structure of the IgA1P/IgA1 complex. The structure of the IgA1P-E1605A/IgA1 complex reveals key details regarding their interaction that includes a gating mechanism that is described further below. Regarding the complex structure, the IgA1P NTD binds to the substrate IgA1 Fab (of the HC) on one end while the IgA1P CTD engages both IgA1 Fc monomers at the other end (Fig. 2b, c). Thus, the stoichiometry of a 1:1 complex can now be understood by the fact that a single IgA1P requires interactions with both IgA1 Fc monomers and therefore prevents two IgA1Ps from symmetrically binding two substrate IgA1s. In fact, weak density can be ascribed to the unbound Fab (Fig. 2a), supporting the fact that despite two IgA1 hinge sites may be available, only one is accessed at a time by IgA1P. The ability of IgA1P to broadly cleave polyclonal IgA1 is also explained by this structure, as IgA1P does not interact with the variable region of the IgA1 substrate (Supplementary Fig. 3).

As the highest resolution information on the complex was found within the active site, most of the IgA1 hinge backbone of the IgA1 hinge could be confidently modeled (Fig. 2d, e and Supplementary Fig. 2d). Following the numbering scheme of the 
a

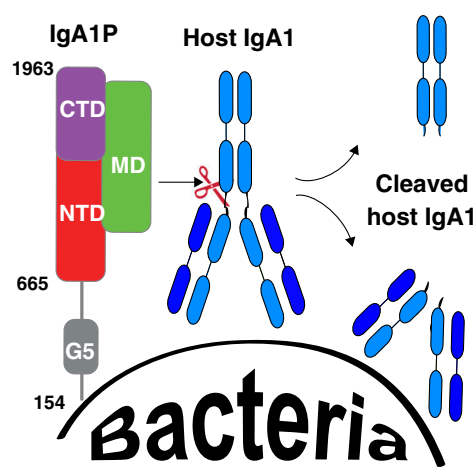

b

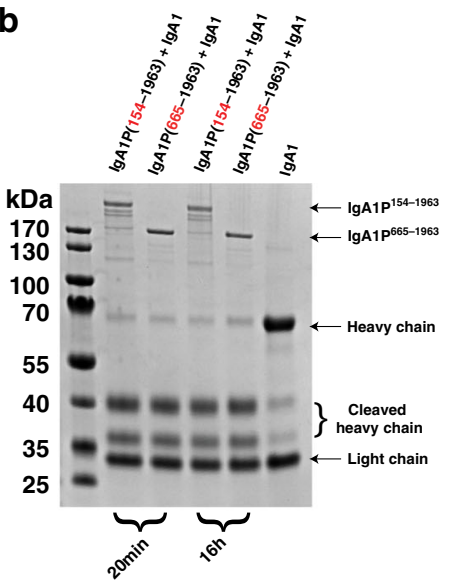

C

d

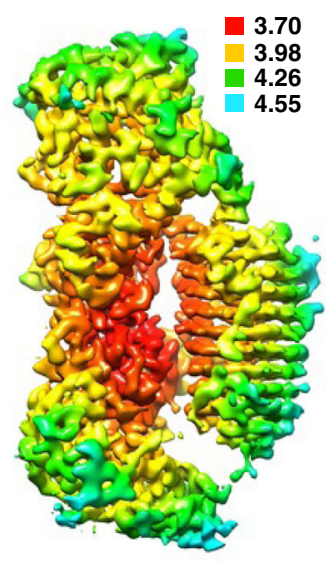

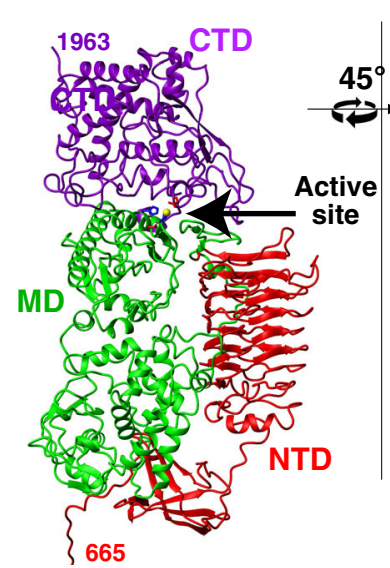

e

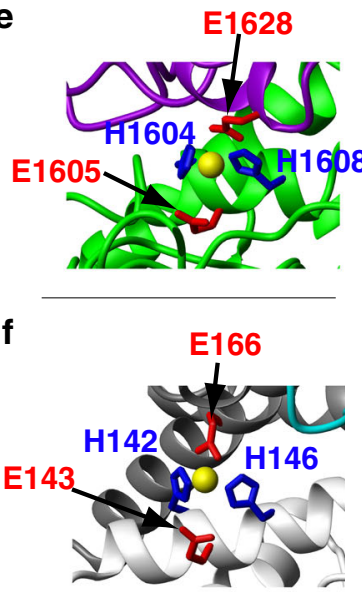

Fig. 1 Function and cryo-EM structure of the S. pneumoniae IgA1P. a The family of IgA1Ps cleave host lgA1 at its hinge region, separating the lgA1 Fc from its Fab and effectively masking bacterial cells with host IgA1 Fab. The general domain architecture of mature S. pneumoniae IgA1P consists of a flexible Nterminal region (residues 154-664) attached to the bacterial cell wall, which includes a small G5 domain followed by a large C-terminal catalytic region (residues 665-1963) ${ }^{21,22}$. b S. pneumoniae IgA1P cleavage is similar between the mature IgA1P 154-1963 and isolated catalytic region of IgA1P 665-1963. Shown is one of two independent SDS-PAGE gel measurements. c 3D reconstruction of S. pneumoniae IgA1P (residues 665-1963) is colored according to the local resolution estimates (units in $\AA$ ). The map was produced using Chimera ${ }^{38}$. d S. pneumoniae IgA1P ribbon structure highlights the tertiary structure (PDB ID: 6XJB). Domains are each color-coded along with the modeled $\mathrm{Zn}$ ion placed based on the superposition of catalytic residues with those found in thermolysin (yellow, arrow). The structure was modeled using Coot $^{32}$. e Expansion of the S. pneumoniae IgA1P active site with H1604, E1605, H1608, and E1628 shown. $\mathbf{f}$ Expansion of the thermolysin active site in a similar orientation to panel E (PDB ID: 1TLX). Functionally homologous thermolysin and IgA1P catalytic residues have the same color coding between panels $\mathbf{e}$ and $\mathbf{f}$.

initial fit IgA1 Fab and Fc models 26,27 , the HC comprising residues 1-221 within the initial Fab model could be extended through the active site of IgA1P to residue S232 (Fig. 2d). Although there is no visible density from residues 233-240, which connects the remaining hinge within the $\operatorname{IgA} 1 \mathrm{HC}$, it is clear that IgA1 residues P227 and T228 are properly aligned for cleavage within the IgA1P cleavage site consistent with previous biochemical studies ${ }^{28}$. Thus, we were able to capture the intact active site within the active complex.

The IgA1P gating mechanism. Potentially the most striking finding of the $S$. pneumoniae IgA1P/IgA1 complex is the conformational change associated with substrate binding (Fig. 3), which underlies an active-site gating mechanism. This large-scale movement is best illustrated by a superposition of the $S$. pneumoniae IgA1P free and bound states (Fig. 3a), which results in a 7.2 $\AA$ RMSD for the whole enzyme but only a $1.5 \AA$ RMSD when the NTD $\beta$-helix is excluded from the RMSD calculation. Thus, it is the NTD that repositions from a "closed" state in the absence of substrate to an "open" state allowing for binding of the IgA1 hinge region. In order to facilitate this domain rearrangement, two flexible loops are involved: IgA1P residues $770-783$ and a $66-$ residue linker that connects the IgA1P NTD to the MD (residues 1051-1116). These loops act as flexible tethers to the entire $\beta$ helix (779-1050), providing enough slack to allow for a shift of the $\beta$-helix by approximately $10 \AA$ relative to the rest of the protease (Fig. 3b).

The number of interactions between the IgA1P NTD and MD are diminished upon this rearrangement, providing for an energetic rationale for why the enzyme "snaps" back after product release. Such findings also led us to hypothesize that the IgA1P NTD may be soluble alone, similar to its G5 domain and CTD that have been previously shown to be independently folded $^{21,22}$. Indeed, we were able to engineer an NTD construct that gives rise to a well-dispersed ${ }^{15} \mathrm{~N}$-HSQC analogous to the independently folded IgA1P CTD and the G5 domain (Supplementary Fig. 4), supporting our findings that the NTD is capable of decoupling from the MD during substrate binding. Thus, $S$. pneumoniae IgA1P exhibits an active-site-gating mechanism in which the NTD $\beta$-helix plays a crucial role. Interestingly, a gating 
a

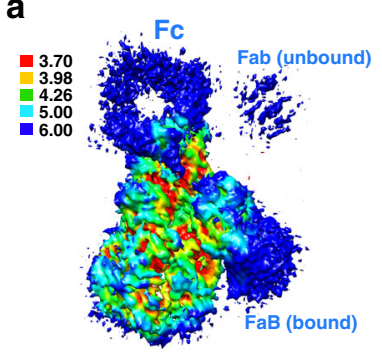

d

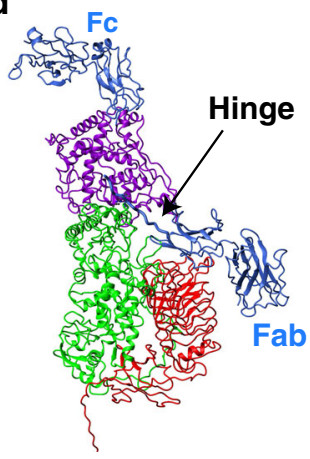

b

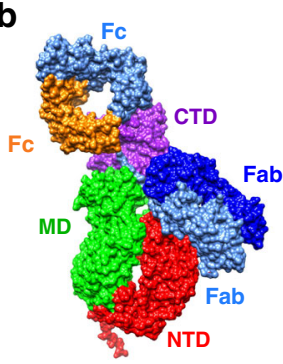

C

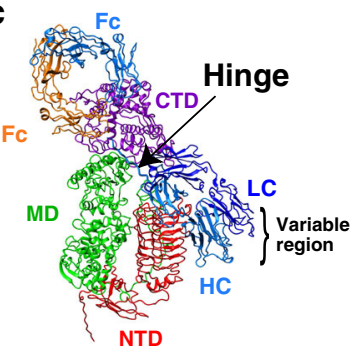

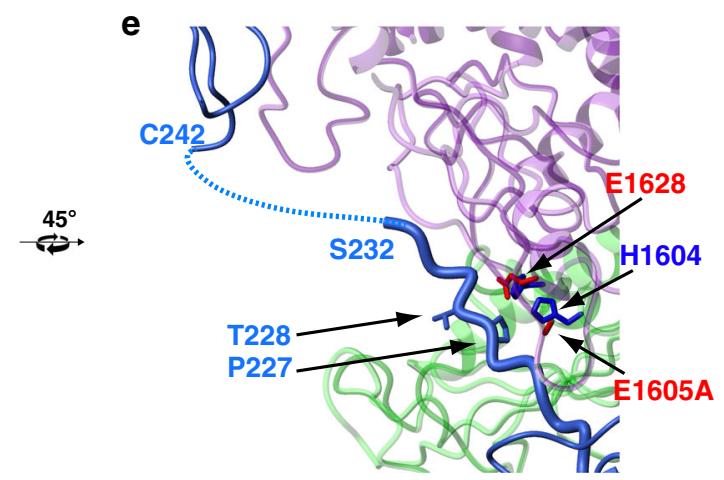

Fig. 2 Cryo-EM structure of the S. pneumoniae IgA1P/IgA1 complex. a 3D reconstruction of S. pneumoniae IgA1P-E1605A in complex with IgA1 is colored according to local resolution estimates (units in $\AA$ ). The map was produced using Chimera ${ }^{38}$. b Surface representation of the IgA1P/IgA1 complex (PDB ID: 6XJA). The model comprises IgA1P residues 665-1963 (domains colored as in Fig. 1d), the IgA1 LC (dark blue), the IgA1 HC portion of the Fab (light blue), and the IgA1 Fc (orange and light blue for each HC monomer). c Ribbon structure of the IgA1P/IgA1 complex. The hinge connecting HC Fab and Fc is indicated by the arrow. $\mathbf{d}$ The IgA1P-IgA1 complex is shown with the bound $\mathrm{HC}$ monomer (both Fab and Fc). e Expansion of the active site with the bound IgA1 hinge. IgA1P catalytic residues are shown according to the colors in Fig. 1e. The scissile bond is between P227 and T228 (residues shown in blue).

a

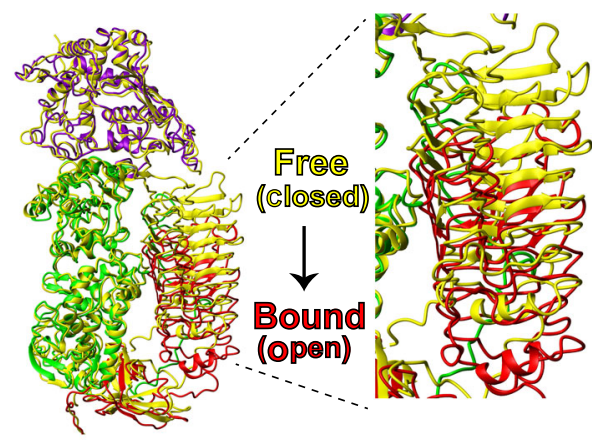

b

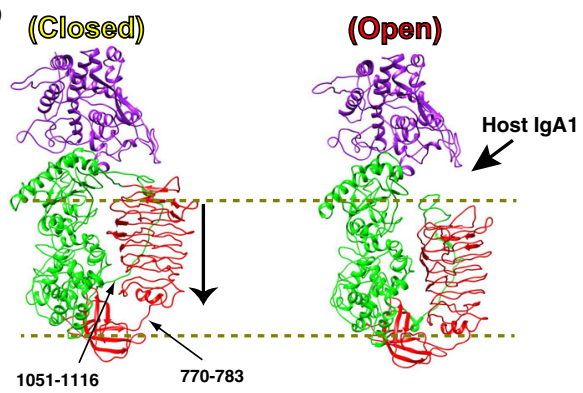

Fig. 3 Structural rearrangement of IgA1P upon IgA1 binding.

a Superposition of IgA1P in both free (yellow) and bound (colored by domain as in Fig. 1d) states. A least squares superposition of IgA1P residues 1115-1963 was used for all C-alpha atoms, illustrating the large $10 \AA$ shift of the entire NTD $\beta$-helix. The unbound state is referred to as "closed" and the bound state as "open". b The $10 \AA$ shift of the NTD $\beta$-helix is illustrated by both the free (left, closed state) and bound IgA1P (right, open state). mechanism has been proposed for the evolutionarily distinct serine-type IgA1P from Haemophilus influenzae $\operatorname{IgA1}^{24}$, but our structure of the $S$. pneumoniae IgA1P/IgA1 complex here provides direct experimental proof that the metalloprotease subgroup of IgA1Ps must undergo such a conformational change to facilitate substrate binding.

Blocking IgA1P active-site-gating through $\mathrm{mAb}$ binding. Considering the clinical potential of utilizing immunogenic regions of IgA1P to develop vaccines preventing $S$. pneumoniae infection 6,7 , we present here evidence validating that such a strategy has the potential to block IgA1P activity. A comparison of the neutralizing activity of a previously developed $\mathrm{mAb}$ used to detect secreted $\operatorname{IgA} 1 \mathrm{P}^{21}$, referred to as $\mathrm{mAb} \# 1$, and a currently produced $\mathrm{mAb}$, referred to as $\mathrm{mAb} \# 2$, reveals that the latter potently blocks IgA1P cleavage of IgA1 and this occurs at stoichiometric concentrations (Fig. 4a). Moreover, this neutralizing mAb also blocks IgA1 substrate binding (Fig. 4b). A 3D reconstruction of IgA1P in the presence of the neutralizing mAb Fab fragment $(\mathrm{mAb} \# 2)$ resulted in a $4.8 \AA$ resolution map (Resolution shells are shown in Fig. $4 c$ and structural parameters and refinement statistics in Supplementary Fig. 2 and Supplementary Table 1), which facilitated an initial rigid body fit of the $\mathrm{mAb} \# 2$ Fab with likely delineation of the individual VL-CL (variable light-constant light) and $\mathrm{VH}-\mathrm{CH} 1$ (variable heavy-constant heavy 1) chains (Supplementary Fig. 5). The final refined model reveals that the neutralizing mAb simultaneously engages both the IgA1P NTD and MD domains (Fig. 4d, e), which confines IgA1P to its closed conformation through intimate contacts with both domains (Fig. 4f, g) and thereby occludes substrate binding. Thus, these studies provide a proof-of-concept that IgA1P activity may be blocked by mAbs. 
a

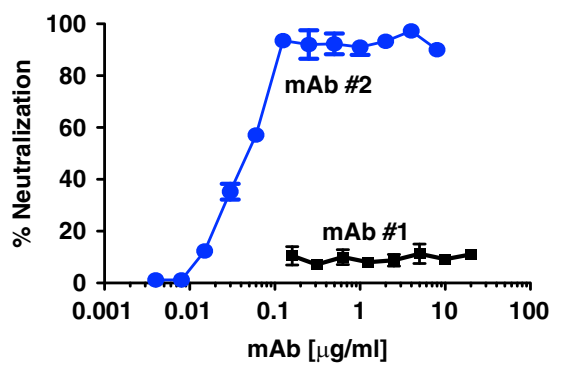

b

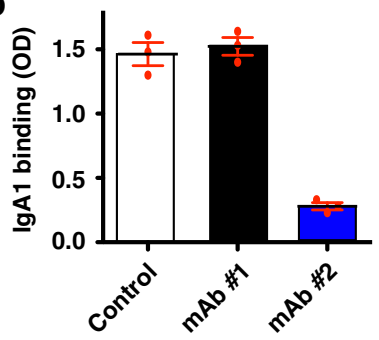

C

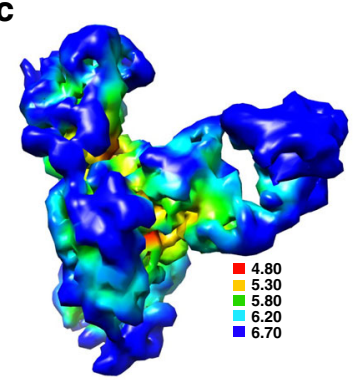

d
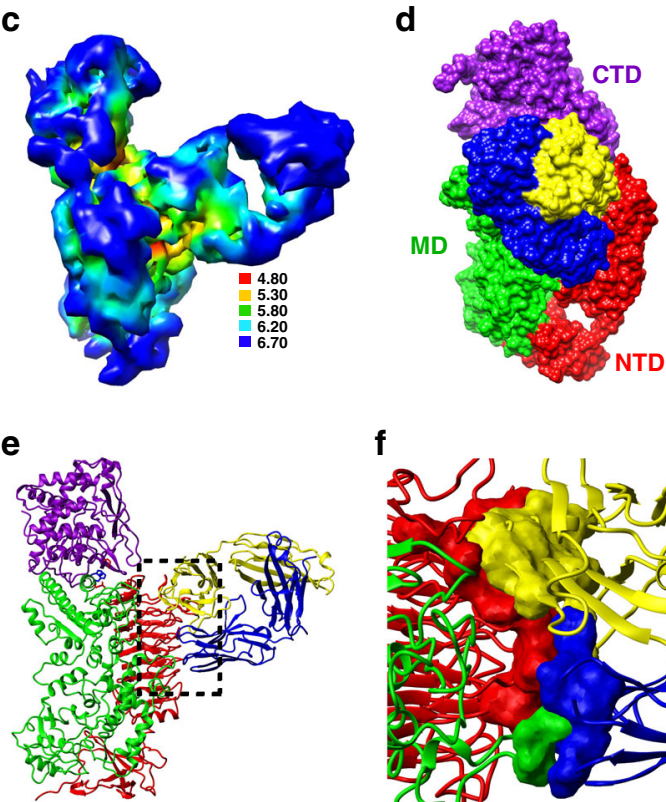

f

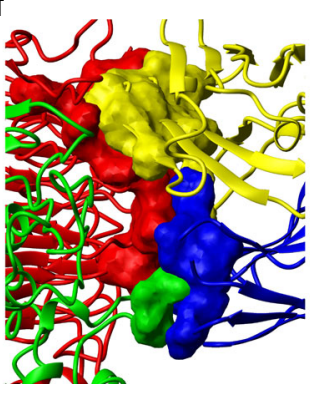

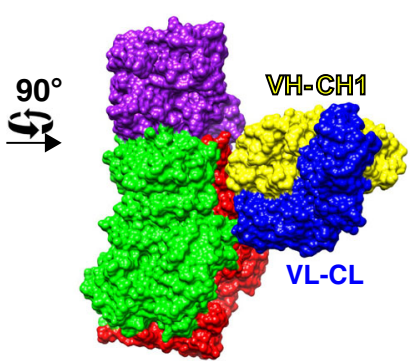

g

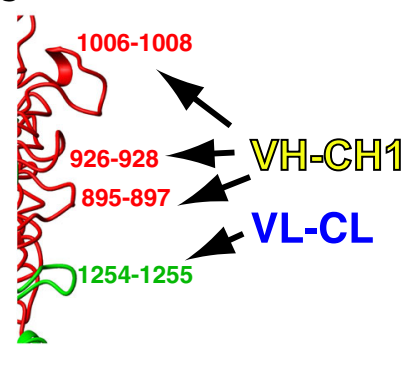

Fig. 4 Cryo-EM structure of an S. pneumoniae IgA1P/mAb complex. a ELISA-based neutralization assay comparing the blocking activity of two mAbs produced against IgA1P to cleave IgA1. The $\mathrm{mAb} \# 1$ (black) previously developed does not neutralize lgA1P activity ${ }^{21}$ and the currently characterized mAb \#2 (blue) does neutralize IgA1P activity. IgA1P residues $154-1963$ was incubated at $286 \mathrm{ng} / \mathrm{ml}(1.3 \mathrm{nM})$ with varying concentrations of the mAbs. For reference, $1 \mu \mathrm{g} / \mathrm{ml}$ of each $\mathrm{mAb}$ is approximately $6.7 \mathrm{nM}$ estimating the molecular weight as $150 \mathrm{kDa}$. Data are an average of $n=4$ biological replicates with the standard error of the mean also shown. b S. pneumoniae IgA1P-E1605A binds to IgA1 (white), which is not blocked by mAb \#1 (black) but is blocked by mAb \#2 (blue). IgA1P-E1605A was plated at $1 \mu \mathrm{g} / \mathrm{ml}$ and incubated with either buffer or $2 \mu \mathrm{g} / \mathrm{ml} \mathrm{mAb}$. Data are an average of $n=3$ biological replicates shown as a dot blot along with the standard error of the mean also shown. c 3D reconstruction of the S. pneumoniae IgA1P in complex with IgA1 colored according to local resolution estimates (units in $\AA$ ). d Surface representation of the $\mathrm{IgA1P} / \mathrm{mAb}$ complex (PDB ID: 7JGJ) with IgA1P shown in a similar orientation as Fig. 1 (left) and rotated $90^{\circ}$ (right). The complex comprises IgA1P (colored as in Fig. 1) and both the mAb VL-CL (blue) and mAb VH$\mathrm{CH} 1$ (yellow). e Ribbon structure of the $\lg A 1 \mathrm{P} / \mathrm{mAb}$ complex with the box highlighting the interaction regions. $\mathbf{f}$ Expansion of the interaction site with residues that form a contact surface within $3 \AA$ shown. $\mathbf{g}$ Specific interacting loops of IgA1P targeted by the mAb are shown. Source data for panels $\mathbf{a}$ and $\mathbf{b}$ are provided as a Source Data File.

\section{Discussion}

We have determined the structure of a member of the M26 metalloprotease IgA1P subfamily of bacterial IgA1Ps both in isolation as well as in complex with its human IgA1 substrate and solved its structure with a neutralizing $\mathrm{mAb}$ that provides insight into blocking $S$. pneumoniae IgA1P activity. Other than the IgA1P NTD that comprises a $\beta$-helix identified in several bacterial proteins $^{23}$, both the IgA1P MD and CTD exhibit no structural similarity to any other previously solved proteins, much less any other metalloprotease. Despite this architecture, catalytic residues contributed by both the IgA1P MD and CTD that include H1604, E1605, H1608, and E1628 are directly comparable in their structural positioning to the same active site residues within the prototypical metalloprotease, thermolysin ${ }^{25}$. The most striking discovery here is how the modular nature of this unique IgA1P facilitates a conformational change that acts as an active-site gate. Specifically, this conformational change can be described as "closed" for the free enzyme and "open" to allow for
IgA1 substrate binding, whereby the mAb occludes substrate binding by binding the IgA1P in the "closed" conformation (Fig. 5). Our studies therefore provide the underlying mechanism by which this unique class of IgA1Ps engages IgA1 and reveals how IgA1Ps can broadly engage IgA1 through contacts with the constant region of the substrate IgA1 HC.

The complex structure with IgA1 also provides an understanding for how IgA1P can cleave the secreted forms of IgA1 (sIgA1) that are joined by the J-chain. Specifically, the Fc portions of each $\operatorname{IgA} 1$ that are coupled by the J-chain leave the IgA1 Fc binding site for IgA1P accessible for cleavage, as shown here by superimposing the bound IgA1 Fc within the IgA1P/IgA1 complex onto the recently solved structure of one of the sIgA1 Fc regions (Fig. 5) ${ }^{29}$.

Considering the sequence similarity of the $S$. pneumoniae IgA1P to many other proteins found within invasive bacteria and across multiple strains (Supplementary Fig. 6a), these studies provide an important foundation for understanding how multiple 


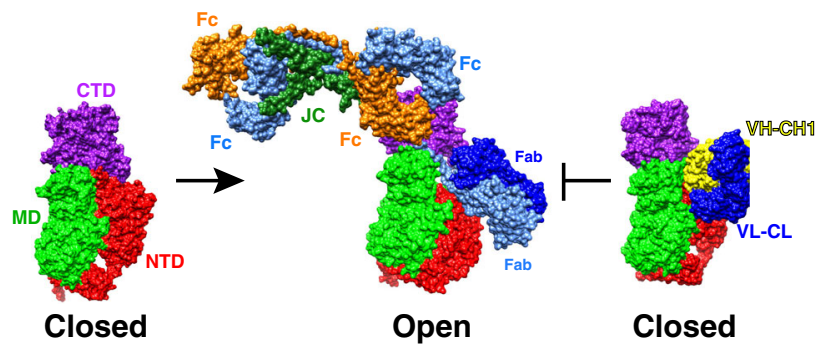

Fig. 5 The structural basis of IgA1P cleavage and mAb neutralization. Models derived from all three cryo-EM reconstructions solved here are shown. Left: IgA1P residues 665-1963 alone (PDB accession 6XJB). Middle: The structure of IgA1P-E1605A in complex with human IgA1 is shown (PDB accession $6 \mathrm{XJA}$ ). The Fc portion of this structural model is superimposed on one of the Fc regions within the recently solved cryo-EM structure of the slgA1 dimer ${ }^{29}$. The J-chain (JC) linking the two Fc portions of slgA1 is also shown (dark green). Right: IgA1P bound to the neutralizing mAb is shown in a similar orientation to illustrate how this mAb occludes substrate binding.

opportunistic pathogens that employ similar IgA1 metalloproteases cleave host IgA1 (Supplementary Fig. 6b). While our cryoEM models demonstrate the specific site of the current neutralizing $\mathrm{mAb}$ is not completely conserved across such strains, there are several highly conserved epitopes that could serve for the creation of broad-spectrum vaccines or multiple exposed epitopes can now be developed to block multiple strains. Lastly, the similarity in the predicted domain structure of this IgA1P in comparison to proteases in the $\mathrm{ZmpB}$ and $\mathrm{ZmpC}$ classes of secreted metalloproteases suggests that the structural data provided here can generally inform upon the mechanisms of cleavage mediated by these related enzymes with possess divergent substrate selectivity ${ }^{30}$.

\section{Methods}

Protein expression and purification. All S. pneumoniae IgA1P constructs of the full catalytic region were engineered and purified similar to that previously described for the mature IgA1P (residues 154-1963), with the exception that the MBP and $6 x H i s$ tags were swapped from their original positions ${ }^{21}$. The kanamycinresistant plasmid, pJ401 (DNA2.0/Atum), was used as the backbone with the open reading frame coding for an $\mathrm{N}$-terminal $\mathrm{MBP}$, a thrombin cleavage site, IgA1P residues 665-1963 (or variants thereof described in the main text), and a Cterminal $6 x$ His tag. A typical growth comprised $4 \mathrm{~L}$ of Luria Broth (LB). Protein was expressed in E. coli BL21(DE3) and induced at an $\mathrm{OD}_{600}$ of $0.6-0.9$ with $1 \mathrm{mM}$ IPTG at $37^{\circ} \mathrm{C}$ for $3 \mathrm{~h}$ before harvesting. Cells were lysed via sonication and lysed supernatants were applied to amylose resin using amylose buffer $(20 \mathrm{mM}$ Tris, $\mathrm{pH}$ 7.4 , and $200 \mathrm{mM} \mathrm{NaCl}$ ) on an AKTA prime FPLC system (GE Healthcare). The protein was eluted using $20 \%$ glucose in amylose buffer, applied to Ni-affinity resin (Sigma) using Ni buffer ( $50 \mathrm{mM}$ phosphate buffer, $\mathrm{pH} 7.5,500 \mathrm{mM} \mathrm{NaCl}$, and $10 \mathrm{mM}$ imidazole), and eluted using Ni buffer supplemented with $0.4 \mathrm{M}$ imidazole. The eluent was then concentrated, cleaved with thrombin (Sigma) at room temperature overnight, and diluted for reapplication to amylose resin to strip remaining $\mathrm{MBP}$ or uncleaved fusion. Finally, amylose-resin flow through was concentrated and applied onto an S200 size exclusion column $(120 \mathrm{ml}$, GE Healthcare) equilibrated in $20 \mathrm{mM}$ HEPES, pH 7.4, and $150 \mathrm{mM} \mathrm{NaCl}$.

The IgA1P G5 domain and CTD were expressed and purified as previously described $^{22}$. The IgA1P NTD was engineered into the pJ401 plasmid and expressed and purified using the same method previously described ${ }^{21,22}$ : an N-terminal $6 \mathrm{xHis}$ tag was followed by a thrombin cleavage site and IgA1P residues 665-1010. All individual domains were purified over $\mathrm{Ni}$-affinity resin, concentrated for thrombin cleavage of their $\mathrm{N}$-terminal $6 \mathrm{xHis}$ tag, cleaved with thrombin at room temperature overnight, and applied to a S75 size exclusion column (120 ml, GE Healthcare).

IgAl kappa was purchased commercially (myBioscience; San Diego, CA) and further purified over a preparative S200 size exclusion column in $20 \mathrm{mM}$ HEPES, $\mathrm{pH} \mathrm{7.4}$, and $150 \mathrm{mM} \mathrm{NaCl}$ in order to specifically select for monomeric IgA1.

The murine monoclonal antibodies used here $(\mathrm{mAb} \# 2)$ and previously described $(\mathrm{mAb} \# 1)^{21}$ were produced as follows. Balb $\mathrm{C}$ female mice were immunized intraperitoneally with $100 \mu \mathrm{g}$ of purified full-length protease with Freund's adjuvant twice and boosted with protein alone at 3 weeks. Proteasespecific IgG in sera collected 7 days after the third injection was detected by ELISA as described below. Spleen cells were fused with PEG and SP2/0 myeloma cells. Hybridomas producing protease-specific IgG were subcloned twice and IgG purified on Protein G columns (Pierce-ThermoFisher; Waltham, MA). Clones reactive with the protease by ELISA were screened and selected based on optimal neutralization as described below (neutralizing activity per $\mu \mathrm{g}$ of protease-specific $\mathrm{IgG}$ ). The Fab fragment for $\mathrm{mAb} \# 2$ used here for cryo-EM in complex with IgA1P residues 665-1963 was excised from the full antibody by use of immobilized Ficin (Thermo Scientific) and diluted using PBS. The "Standardized Protocol for Production of Monoclonal Antibodies in Mice", protocol \# 104513(12)1C, was approved by the Animal Care and Use Committee, accredited by the Association for Assessment and Accreditation of Laboratory Animal Care-File Number 00235 . We have complied with all relevant ethical regulations for animal testing and research.

Cryo-EM sample preparation and data collection. For $S$. pneumoniae IgA1P alone, purified S. pneumoniae IgA1P (residues 665-1963) was concentrated to $\sim 3.4 \mu \mathrm{M}(0.5 \mathrm{mg} / \mathrm{ml})$ in $20 \mathrm{mM}$ HEPES, pH 7.4, and $150 \mathrm{mM} \mathrm{NaCl}$. For the S. pneumoniae IgA1P/IgA1 complex, purified S. pneumoniae IgA1P-E1605A (residues 665-1963) was concentrated to $90 \mu \mathrm{M}$ and was incubated with $30 \mu \mathrm{M}$ of the IgAl substrate in the presence of $1 \mathrm{mM}$ EDTA. This IgA1P/substrate complex was further purified in $20 \mathrm{mM}$ HEPES, pH 7.4, and $150 \mathrm{mM} \mathrm{NaCl}, 1 \mathrm{mM}$ EDTA over an analytical Superdex 200 (GE Healthcare) in order to enrich the 1:1 complex (Supplementary Fig. 1d). The same procedure was used for the S. pneumoniae IgA1P/mAb (the Fab fragment) complex but using wild type IgA1P instead of the IgA1P-E1605A mutant and in the absence of EDTA. For grid preparations, $3 \mu \mathrm{l}$ of $0.1-0.2 \mathrm{mg} / \mathrm{ml}$ sample was applied to plasma-cleaned C-flat holy carbon grids $(1.2 / 1.3,400$ mesh) and frozen using a Vitrobot Mark IV (Thermo Fisher Scientific), with the environmental chamber set at $100 \%$ humidity and $4{ }^{\circ} \mathrm{C}$. The grids were blotted for 2.5-3.0 s and then flash frozen in liquid-nitrogen-cooled liquid ethane. A full description of the cryo-EM data collection can be found in Supplementary Table 1 .

Cryo-EM data processing and structural modeling. Data for the free $S$. pneumoniae IgA1P (residues 665-1963) were processed in RELION ${ }^{31}$ and that of the $S$ pneumoniae IgA1P-E1605A/IgA1 complex were processed in both RELION and cryoSPARC ${ }^{23,31}$. Models for both complexes were built using $\operatorname{Coot}^{32}$. For IgA1P alone, the movies were motion corrected using MotionCor $2^{33}$ and their contrast transfer functions were estimated using $\mathrm{Gctf}^{34}$. Micrographs with fit resolution of worse than $6 \AA$ were discarded. $\sim 1500$ particles were manually picked and classified into 10 classes. Meaningful 5 representative class averages were selected and used for template picking. The particles were extracted with a box size of $336 \times 336$ pixels and subjected to 2D classification. Classes with clear visible secondary features were selected and subjected to ab initio reconstruction, 3D classification, and refinement. After particle polishing and CTF refinement, the final model for unbound IgA1P (residues 665-1963) was estimated as $3.77 \AA$ resolution based on the gold-standard Fourier shell correlation (FSC) measurement. The sequence of IgA1P residues 665-1963 was analyzed by multiple secondary structure prediction servers as well as the I-TASSER 3D structure prediction server ${ }^{35}$. This analysis revealed the NTD comprised the $\beta$-helix domain (795-1050), which served as the starting point of our model building. For the rest of the IgA1P model, the mainchain was traced with the help of MAINMAST ${ }^{36}$. The final model was complete in $\operatorname{Coot}^{32}$, followed by the real-space refinement in PHENIX ${ }^{37}$.

For the IgA1P-E1605A/IgA1 complex, a similar process protocol was carried out, except that the particles were binned twice to make the final pixel size $1.06 \AA$ / px. The resolution of the final reconstruction for the IgA1P-E1605A/IgA1 complex was estimated as $3.5 \AA$ in RELION with similar resolution in cryoSPARC. This map facilitated rigid body fitting using the free IgA1P residues 665-1963 solved above, the substrate IgA1 Fc (PDB accession number 1OW0), and the substrate IgA1 Fab (PDB accession 3M8O), which was further refined (Fig. 2). As the IgA1 used was polyclonal, all variable residues within the Fab region of both its $\mathrm{HC}$ and LC were modeled as alanine residues (Supplementary Fig. 3). This did not alter the model of IgA1P as IgA1P only makes contacts with the IgAl constant region.

For the IgA $1 \mathrm{P} / \mathrm{mAb}$ complex, processing was identical to that of the free protease. The solution of the final reconstruction for the IgA1P/mAb complex was $4.8 \AA$. The free IgA1P residues $674-1963$ and a model of the mAb constructed in SWISS-MODEL was placed within this $3 \mathrm{D}$ reconstruction using Chimera. For this initial mAb model, the mAb HC and LC were sequenced and PDB accession 3RHW and 4HXA were found to be the most similar, respectively. Models for these two chains were produced separately in SWISS-MODEL, superimposed upon that of the full 3RHW Fab, and then placed within the 3D reconstruction within Chimera.

All data were collected with Leginon 3.3. The local resolution estimations of both reconstructions were performed using RELION's own postprocessing protocol and models were refined using both Phenix ${ }^{37}$ and $\operatorname{Coot}^{32}$.

NMR sample preparation and data collection. All NMR data were collected on a Varian 900 equipped with a cryoprobe. Samples were produced as previously described using ${ }^{15} \mathrm{~N}$ M9 minimal media for the S. pneumoniae IgA1P G5 domain (residues $312-393)^{22}$ and ${ }^{15} \mathrm{~N},{ }^{2} \mathrm{H}$ M9 minimal media for both the CTD (residues 1611-1963) ${ }^{21}$ and the NTD (residues 665-1010). Final size exclusion was performed in NMR buffer ( $50 \mathrm{mM} \mathrm{Na}_{2} \mathrm{HPO}_{4}, \mathrm{pH} 6.5$, and $\left.150 \mathrm{mM} \mathrm{NaCl}\right)$. 
Protease neutralization assay. 96 well microtiter plates were coated with antiIgA1 CH3 (catalog number ab17747, Abcam; Cambridge, MA) $(2 \mathrm{mg} / \mathrm{ml})$ overnight at $4{ }^{\circ} \mathrm{C}$, washed, and blocked with PBST-BSA for $2 \mathrm{~h}$ at room temperature. Wild type IgA1P residues $154-1963(286 \mathrm{ng} / \mathrm{ml} ; 1.3 \mathrm{nM})$ was incubated overnight with the indicated doses of the neutralizing $\mathrm{mAb}$ at $4{ }^{\circ} \mathrm{C}$. Human IgAl substrate (catalog number MBS318189, myBioscience; San Diego, CA) alone or with equal volumes of the mAb/IgA1P mixture were incubated for $1 \mathrm{hr}$ at $37^{\circ} \mathrm{C}$ and added to the plates for $2 \mathrm{~h}$ at room temperature. After washing, alkaline phosphatase-labeled goat anti-human Kappa (catalog number A38913, Sigma; St. Louis, MO) or goat HRP-labeled anti-human IgA CH3 (Abcam; Cambridge, MA) was added for an hour at room temperature. Intact or cleaved IgA1 was detected by the presence or absence of bound kappa light chain using alkaline phosphatase-labeled goat antihuman Kappa (Sigma; St. Louis, MO), which is lost by IgA1 cleavage. IgA1 binding was normalized by HRP-labeled goat anti-human Fca. Both are read at $405 \mathrm{~nm}$ in separate wells. Plates were either developed with p-nitrophenyl phosphate substrate-AP (Sigma) or ABTS-HRP, respectively.

IgA1 binding assay. 96 well Nunc Maxisorp plates were coated with $1 \mu \mathrm{g} / \mathrm{ml}$ (4.6 nM) IgA1P-E1605A residues 154-1963 in PBS overnight at $4{ }^{\circ} \mathrm{C}$, blocked for $2 \mathrm{~h}$ with PBS-Tween20-Thimersol-0.5\% BSA(PBS-TT-BSA) and incubated with $100 \mathrm{ng} / \mathrm{ml}$ human IgA1 (myBioscience; San Diego, CA), which was followed by the addition of $2 \mu \mathrm{g} / \mathrm{ml} \mathrm{mAb}(14.6 \mathrm{nM})$. Human IgA1 that bound to the protease was detected with HRP-labeled goat anti-human IgA, developed with peroxide and ABTS, and optical densities measured at $405 \mathrm{~nm}$ with Veramax ELISA plate reader.

Reporting summary. Further information on research design is available in the Nature Research Reporting Summary linked to this article.

\section{Data availability}

The cryo-EM maps are deposited in the Electron Microscopy Data Bank under accession codes "EMD-22205" (IgA1P residues 665-1963), "EMD-22204" (IgA1P residues 665-1963 with the single E1605A mutation in complex with IgA1), "EMD-22328" (IgA1P residues 665-1963 in complex with the mAb). Structure coordinates are deposited at the Protein Data Bank with accession codes "6XJB" (IgA1P residues 665-1963), "6XJA" (IgA1P residues 665-1963 with the single E1605A mutation in complex with IgA1), "7JGJ" (IgA1P residues 665-1963 in complex with the mAb). Source data is provided with this paper.

Received: 11 August 2020; Accepted: 30 October 2020; Published online: 27 November 2020

\section{References}

1. O’Brien, K. L. et al. Burden of disease caused by Streptococcus pneumoniae in children younger than 5 years: global estimates. Lancet 374, 893-902 (2009).

2. Janoff, E. N. \& Musher, D. N. Madell, Doublas, and Bennett's Streptococcus pneumoniae. In Mandell, Gordon, Douglas and Bennett. Principles and Practice of Infectious Disease Vol. e5 Ch. 199, 2473-2491 (Elsevier, 2020).

3. Ramirez, J. A. et al. Adults hospitalized with pneumonia in the United States: incidence, epidemiology, and mortality. Clin. Infect. Dis. 65, 1806-1812 (2017).

4. Jain, S., Self, W. H., Wunderink, R. G. \& Team, C. E. S. Community-acquired pneumonia requiring hospitalization REPLY. N. Engl. J. Med. 373, 2382-2382 (2015).

5. Bonten, M. J. M. et al. Polysaccharide conjugate vaccine against pneumococcal pneumonia in adults. N. Engl. J. Med. 372, 1114-1125 (2015).

6. Janoff, E. N. et al. Pneumococcal IgA1 protease subverts specific protection by human IgA1. Mucosal Immunol. 7, 249-256 (2014).

7. Mehr, S. \& Wood, N. Streptococcus pneumoniae - a review of carriage, infection, serotype replacement and vaccination. Paediatr. Respiratory Rev. 13, 258-264 (2012).

8. Perez-Dorado, I., Galan-Bartual, S. \& Hermoso, J. A. Pneumococcal surface proteins: when the whole is greater than the sum of its parts. Mol. Oral. Microbiol. 27, 221-245 (2012).

9. Poulsen, K. et al. A comprehensive genetic study of streptococcal immunoglobulin A1 proteases: evidence for recombination within and between species. Infect. Immun. 66, 181-190 (1998).

10. Briles, D. E., Paton, J. C., Mukerji, R., Swiatlo, E. \& Crain, M. J. Pneumococcal vaccines. Microbiol. Spectrum 7, https://doi.org/10.1128/microbiolspec.GPP30028-2018 (2019).

11. Ramos-Sevillano, E., Ercoli, G. \& Brown, J. S. Mechanisms of naturally acquired immunity to Streptococcus pneumoniae. Front. Immunol. 10, 358 (2019).
12. Rawlings, N. D. et al. The MEROPS database of proteolytic enzymes, their substrates and inhibitors in 2017 and a comparison with peptidases in the PANTHER database. Nucleic Acids Res. 46, D624-D632 (2018).

13. Bender, M. H. \& Weiser, J. N. The atypical amino-terminal LPNTGcontaining domain of the pneumococcal human IgA1-specific protease is required for proper enzyme localization and function. Mol. Microbiol. 61, 526-543 (2006).

14. Mistry, D. V. \& Stockley, R. A. The cleavage specificity of an IgA1 protease from Haemophilus influenzae. Virulence 2, 103-110 (2011).

15. Mortensen, S. B. \& Kilian, M. Purification and characterization of an immunoglobulin-A1 protease from bacteroides-melaninogenicus. Infect. Immun. 45, 550-557 (1984).

16. Woof, J. M. \& Russell, M. W. Structure and function relationships in IgA Mucosal Immunol. 4, 590-597 (2011).

17. Heineke, M. H. \& van Egmond, M. Immunoglobulin A: magic bullet or Trojan horse? Eur. J. Clin. Investig. 47, 184-192 (2017).

18. Mansa, B. \& Kilian, M. Retained antigen-binding activity of FAB-alpha fragments of human monoclonal immunoglobulin cleaved by igA protease. Infect. Immun. 52, 171-174 (1986).

19. Kilian, M., Mestecky, J. \& Russell, M. W. Defense-mechanisms involving FCdependent functions of immunoglobulins-A and their subversion by bacterial immunoblobuilin-A proteases. Microbiological Rev. 52, 296-303 (1988).

20. Proctor, M. \& Manning, P. J. Production of immunoglobulin-A1 protease by Streptococcus pneumoniae from animals. Infect. Immun. 58, 2733-2737 (1990).

21. Chi, Y. C. et al. Streptococcus penumoniae IgA1 protease: a metalloprotease that can catalyze in a split manner in vitro. Protein Sci. 26, 600-610 (2017).

22. Paukovich, N. et al. Streptococcus pneumoniae G5 domains bind different ligands. Protein Sci. 28, 1797-1805 (2019).

23. Schulz, E. C. \& Ficner, R. Knitting and snipping: chaperones in beta-helix folding. Curr. Opin. Struct. Biol. 21, 232-239 (2011).

24. Johnson, T. A., Qiu, J., Plaut, A. G. \& Holyoak, T. Active-site gating regulates substrate selectivity in a chymotrypsin-like serine protease: the structure of Haemophilus influenzae immunoglobulin Al protease. J. Mol. Biol. 389, 559-574 (2009)

25. Adekoya, O. A. \& Sylte, I. The thermolysin family (M4) of enzymes: therapeutic and biotechnological potential. Chem. Biol. Drug Des. 73, 7-16 (2009).

26. Correa, A. et al. Structure of a human IgA1 Fab fragment at 1.55 angstrom resolution: potential effect of the constant domains on antigen-affinity modulation. Acta Crystallogr. Sect. D.-Struct. Biol. 69, 388-397 (2013).

27. Posgai, M. T. et al. Fc alpha RI binding at the IgA1 $\mathrm{C}(\mathrm{H}) 2-\mathrm{C}(\mathrm{H}) 3$ interface induces long-range conformational changes that are transmitted to the hinge region. Proc. Natl Acad. Sci. USA 115, E8882-E8891 (2018).

28. Senior, B. W. \& Woof, J. M. The influences of hinge length and composition on the susceptibility of human IgA to cleavage by diverse bacterial IgAl proteases. J. Immunol. 174, 7792-7799 (2005).

29. Kumar, N., Arthur, C. P., Ciferri, C. \& Matsumoto, M. L. Structure of the secretory immunoglobulin A core. Science 367, 1008 (2020).

30. Bek-Thomsen, M., Poulsen, K. \& Kilian, M. Occurrence and evolution of the paralogous zinc metalloproteases IgAl protease, $\mathrm{ZmpB}, \mathrm{ZmpC}$, and $\mathrm{ZmpD}$ in Streptococcus pneumoniae and related commensal species. Mbio 3, e00303 (2012).

31. Scheres, S. H. W. in Resolution Revolution: Recent Advances in Cryoem Vol. 579 Methods in Enzymology (ed Crowther, R. A.) 125-157 (2016).

32. Emsley, P., Lohkamp, B., Scott, W. G. \& Cowtan, K. Features and development of Coot. Acta Crystallogr. Sect. D.-Biol. Crystallogr. 66, 486-501 (2010).

33. Zheng, S. Q. et al. MotionCor2: anisotropic correction of beam-induced motion for improved cryo-electron microscopy. Nat. Methods 14, 331-332 (2017).

34. Zhang, K. Gctf: Real-time CTF determination and correction. J. Struct. Biol. 193, 1-12 (2016).

35. Zheng, W., Zhang, C. X., Bell, E. W. \& Zhang, Y. I-TASSER gateway: a protein structure and function prediction server powered by XSEDE. Future Gener. Computer Syst.- Int. J. Escience 99, 73-85 (2019).

36. Perez, A. et al. MAINMAST-MELD-MDFF: denovo structure-determination with data-guided molecular dynamics. Biophysical J. 116, 287A-288A (2019)

37. Adams, P. D. et al. PHENIX: a comprehensive Python-based system for macromolecular structure solution. Acta Crystallogr. Sect. D.-Biol. Crystallogr. 66, 213-221 (2010).

38. Pettersen, E. F. et al. UCSF chimera - A visualization system for exploratory research and analysis. J. Computational Chem. 25, 1605-1612 (2004).

\section{Acknowledgements}

We thank the CU Cryo-EM Structural Biology Shared Resource Facility for data collection for IgA1P alone. We would like to thank Lori Sherman and the University of Colorado Cancer Center Protein Production/MoAB/Tissue Culture Shared Resource for their help 
with monoclonal antibody production. Data collection of the IgA1P-E1605A/IgA1 complex was supported by NIH grant U24GM129547 and performed at the PNCC at OHSU and accessed through EMSL (grid.436923.9), a DOE Office of Science User Facility sponsored by the Office of Biological and Environmental Research. NMR spectra were collected at the University of Colorado Spectroscopy Facility supported by the University of Colorado Cancer Center NIH P30 CA046934. J.E. was supported by Veterans Affairs Research Service I01BX004320 and NIH R21 AI092468. H.Z. was supported by NIH R01 GM126626. E.Z.E. was supported by NIH R21 AI146295 and NSF application number 1807326.

\section{Author contributions}

Z.W. and H.Z. determined the 3D reconstructions and Z.W. refined IgA1P alone. N.T., T.H. and E.Z.E. refined both complexes. Y.C.C. began the initial cloning of the IgA1P with E.Z.E., J.R. and E.J. produced the mAb with J.R. and E.Z.E. purifying the product. J.S.R. and E.Z.E. purified all proteins and complexes.

\section{Competing interests}

The authors declare no competing interests.

\section{Additional information}

Supplementary information is available for this paper at https://doi.org/10.1038/s41467020-19887-3

Correspondence and requests for materials should be addressed to H.Z., E.J. or E.E.
Peer review information Nature Communications thanks Daniel Lightwood, Christian Spoerry and Junyu Xiao for their contribution to the peer review of this work. Peer reviewer reports are available.

Reprints and permission information is available at http://www.nature.com/reprints

Publisher's note Springer Nature remains neutral with regard to jurisdictional claims in published maps and institutional affiliations.

(c) Open Access This article is licensed under a Creative Commons Attribution 4.0 International License, which permits use, sharing, adaptation, distribution and reproduction in any medium or format, as long as you give appropriate credit to the original author(s) and the source, provide a link to the Creative Commons license, and indicate if changes were made. The images or other third party material in this article are included in the article's Creative Commons license, unless indicated otherwise in a credit line to the material. If material is not included in the article's Creative Commons license and your intended use is not permitted by statutory regulation or exceeds the permitted use, you will need to obtain permission directly from the copyright holder. To view a copy of this license, visit http://creativecommons.org/ licenses/by/4.0/.

(C) The Author(s) 2020 